\title{
新型含噻唑环的吡唑肜醚类衍生物的合成及其生物活性
}

\author{
戴 红*,a,b 于海波 ${ }^{a}$ 刘建兵 ${ }^{a}$ 秦 雪 ${ }^{a}$ \\ 王婷婷 ${ }^{a}$ 张 欣 ${ }^{a}$ 秦振芳 ${ }^{a}$ 方建新 $*, a$
}

(南开大学元素有机化学国家重点实验室 天津 300071)

(南通大学化学化工学院 南通 226019)

\begin{abstract}
摘要 为了寻找活性较好的杂环农药, 将噻唑基团引入到吡唑分子结构中, 设计合成了一系列新型含噻唑环的吡唑朊 醚衍生物. 通过 ${ }^{1} \mathrm{H} \mathrm{NMR} 、{ }^{13} \mathrm{C} \mathrm{NMR}$ 和元素分析测试对标题化合物的结构进行了表征. 对合成的目标化合物进行了初步 的生物活性测试, 部分化合物显示出一定的的杀菌、杀虫或植物生长调节活性.

关键词 吡唑肜; 噻唑; 合成; 生物活性
\end{abstract}

\section{Synthesis and Bioactivities of Novel Pyrazole Oxime Ether Deriva- tives Containing a Thiazolyl Moiety}

\author{
Dai, Hong $^{*, a, b} \quad$ Yu, Haibo $^{a} \quad$ Liu, Jianbing $^{a} \quad$ Qin, Xue $^{a} \quad$ Wang, Tingting $^{a}$ \\ Zhang, Xin ${ }^{a} \quad$ Qin, Zhenfang ${ }^{a} \quad$ Fang, Jianxin*,a \\ ( ${ }^{a}$ State Key Laboratory of Elemento-organic Chemistry, Nankai University, Tianjin 300071) \\ ( ${ }^{b}$ College of Chemistry and Chemical Engineering, Nantong University, Nantong 226019)
}

\begin{abstract}
In order to find potent pesticide containing heterocycle, a series of novel pyrazole oxime ether derivatives containing a thiazolyl moiety were designed and synthesized by introducing the significant thiazole ring into the molecular structure of pyrazole. The structures of these compounds were characterized by ${ }^{1} \mathrm{H}$ NMR, ${ }^{13} \mathrm{C}$ NMR spectra and elemental analysis. Bioactivities of the target compounds were performed, and some compounds exhibited certain fungicidal, insecticidal or plant growth regulatory activities.
\end{abstract}

Keywords pyrazole oxime; thiazole; synthesis; biological activity

杂环化合物是当前新药发展的主流, 而在杂环化合 物中则以含氮杂环为主. 吡唑类化合物作为含氮杂环体 系中重要一员, 在农药和医药领域都表现出较好的生物 活性, 如杀菌 ${ }^{[1 \sim 4]}$ 、除草 ${ }^{[5]}$ 、杀虫 ${ }^{[6,7]}$ 、植物生长调节 ${ }^{[8]}$ 及抗癌的等, 因而颇受人们的青睐. 噻唑类化合物亦是 一类重要的含氮杂环化合物, 具有杀虫 ${ }^{[10]}$ 、除草 ${ }^{[11]}$ 、杀 菌 ${ }^{[12,13]}$ 、抗炎 ${ }^{[14]}$ 及抗肿瘤 ${ }^{[15,16]}$ 等生物活性, 在人类保健 和农业生产中扮演着十分重要的角色, 并已成为药物合 成研究的一个热点. 目前, 国内外药物化学公司相继成 功开发了含噻唑环结构的农药新品种, 如杀虫剂噻虫 胺 ${ }^{[17]}$ 、杀菌剂苯噻菌胺 ${ }^{[18]}$ 、抗植物病毒剂毒氟磷 ${ }^{[199}$ 等.
此外，近年来肟醚衍生物也以其良好的生物活性及独特 的结构特征而受到化学家的普遍关注 ${ }^{[20 ~ 22]}$, 肜醚结构 常作为良好的药效团引入到母体化合物分子中，以改善 其生物活性. 为了寻找新的含吡唑杂环的先导化合物, 我们采用活性基团拼接的方法，设计合成了一系列未见 文献报道的新型含噻唑环的吡唑肜醚衍生物. 通过 ${ }^{1} \mathrm{H}$ $\mathrm{NMR},{ }^{13} \mathrm{C} \mathrm{NMR}$ 和元素分析等方法对目标化合物的结构 进行了表征. 目标化合物的合成路线如 Scheme 1 所示. 对所合成的化合物进行了初步的生物活性测试，结果表 明，部分标题化合物具有一定的杀菌、杀虫或植物生长 调节活性.

\footnotetext{
*E-mail: daihong_2001@yahoo.com.cn,yssfjx@yahoo.com.cn

Received November 27, 2012; revised January 6, 2013; published online January 11, 2013.

Project supported by the National Natural Science Foundation of China (Nos. 20772068, 21202089) and the Scientific Research Foundation for Talent Introduction of Nantong University (No. 12R036).

国家自然科学基金(Nos. 20772068, 21202089)及南通大学引进人才基金(No. 12R036)资助项目.
} 


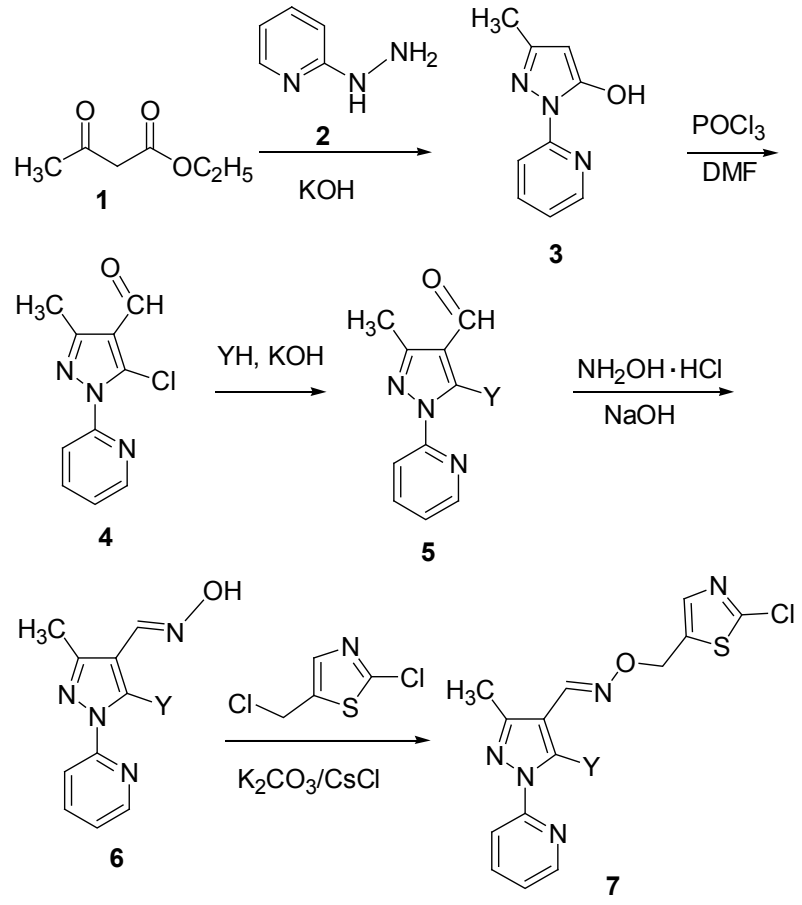

7a: $\mathrm{Y}=\mathrm{C}_{6} \mathrm{H}_{5} \mathrm{O}, 7 \mathbf{b}: \mathrm{Y}=4-\mathrm{ClC}_{6} \mathrm{H}_{4} \mathrm{O}, 7 \mathbf{c}: \mathrm{Y}=6-\mathrm{Cl}-3-\mathrm{CH}_{3} \mathrm{C}_{6} \mathrm{H}_{3} \mathrm{O}$, 7d: $Y=1 H$-pyrazolyl, 7e: $Y=1 H$-1,2,4-triazolyl,

7f: $Y=1$-piperidinyl, 7g: $Y=1$-morpholinyl

Scheme 1

\section{1 实验部分}

\section{1 仪器与试剂}

熔点仪为北京泰克仪器有限公司, X-4 型数字显微 熔点仪, 未经校正; Bruker AC-P 300 型核磁共振仪, 以 $\mathrm{CDCl}_{3}$ 为溶剂, $\mathrm{TMS}$ 为内标; 柱层析采用柱层析硅胶 (200 300 目)(青岛海洋化工厂). 所用试剂均为市售分 析纯.

\section{2 合成}

\subsection{1 中间体的合成}

1-(2-吡啶基)-3-甲基-5-差基吡唑(3)按文献[23]方法 制备，1-(2-吡啶基)-3-甲基-5-氯吡唑-4-甲醛(4)按文献 [24]方法制备, 中间体 1-(2-吡啶基)-3-甲基-5-芳氧基(或 杂环基)吡坐-4-甲醛(5)和 1-(2-吡啶基)-3-甲基-5-芳氧基 (或杂环基)吡唑-4-甲酰基肜(6)按文献[25]方法制备.

1.2.21-(2-吡啶基)-3-甲基-5-芳氧基(或杂环基)吡唑4-甲酰基- $O$-[(2-氯噻唑-5-基)甲基]肜(7)的合成通法

在 $100 \mathrm{~mL}$ 四口瓶中, 加入 $2.5 \mathrm{mmol}$ 肜中间体 6、 $2.8 \mathrm{mmol} 2$-氯-5-氯甲基噻唑、 $8 \mathrm{mmol}$ 无水碳酸钾及 30 $\mathrm{mL}$ 丙酩, 室温摚拌 $10 \mathrm{~min}$, 加入催化量的氯化铯, 加热 回流 $10 \sim 12$ h. 冷却至室温, 浓缩反应液, 残余物用 80 $\mathrm{mL}$ 乙酸乙酯溶解, 依次用水和 $10 \% \mathrm{Na}_{2} \mathrm{CO}_{3}$ 溶液洗涤, 无水 $\mathrm{MgSO}_{4}$ 干燥. 抽滤, 减压蒸除溶剂, 残余物以石油 醚/乙酸乙酯 $(V: V=7: 1)$ 为淋洗剂, 通过硅胶 $(200 \sim$
300 目)柱层析分离得到纯品 $7 \mathbf{a} \sim 7 \mathbf{g}$.

1-(2-吡啶基)-3-甲基-5-苯氧基吡唑-4-甲酰基- $O$-[(2氯噻唑-5-基)甲基]肟(7a): 黄色油状物, 收率 64.2\%. ${ }^{1} \mathrm{H}$ NMR $\left(\mathrm{CDCl}_{3}, 300 \mathrm{MHz}\right) \delta: 8.38(\mathrm{~d}, J=3.9 \mathrm{~Hz}, 1 \mathrm{H}, \mathrm{PyH})$, $7.85(\mathrm{~s}, 1 \mathrm{H}, \mathrm{CH}=\mathrm{N}), 7.63 \sim 7.71(\mathrm{~m}, 2 \mathrm{H}, \mathrm{PyH}), 7.41(\mathrm{~s}$, $1 \mathrm{H}, \mathrm{C}=\mathrm{CH}), 7.26(\mathrm{t}, J=7.5 \mathrm{~Hz}, 2 \mathrm{H}, \mathrm{ArH}), 7.04 \sim 7.13(\mathrm{~m}$, 2H, ArH, PyH), 6.93 (d, J=8.1 Hz, 2H, ArH), 5.08 (s, 2H, $\left.\mathrm{CH}_{2}\right), 2.53\left(\mathrm{~s}, 3 \mathrm{H}, \mathrm{CH}_{3}\right) ;{ }^{13} \mathrm{C} \mathrm{NMR}\left(\mathrm{CDCl}_{3}, 75 \mathrm{MHz}\right) \delta$ : $157.0,152.8,150.1,149.2,148.9,148.1,141.6,140.3$, $138.4,136.8,129.8,123.6,122.2,115.8,115.6,102.8$, 67.5, 15.4. Anal. calcd for $\mathrm{C}_{20} \mathrm{H}_{16} \mathrm{ClN}_{5} \mathrm{O}_{2} \mathrm{~S}$ : C 56.40, $\mathrm{H}$ 3.79, N 16.44, found C 56.29, H 3.66, N 16.30.

1-(2-吡啶基)-3-甲基-5-(4-氯苯氧基)吡唑-4-甲酰基$O$-[(2-氯噻唑-5-基)甲基]肟(7b): 淡黄色固体, 收率 70.1\%. m.p. 91 93 ${ }^{\circ} \mathrm{C}$; ${ }^{1} \mathrm{H}$ NMR $\left(\mathrm{CDCl}_{3}, 300 \mathrm{MHz}\right) \delta$ : $8.36(\mathrm{~d}, J=4.8 \mathrm{~Hz}, 1 \mathrm{H}, \mathrm{PyH}), 7.86(\mathrm{~s}, 1 \mathrm{H}, \mathrm{CH}=\mathrm{N})$, $7.72 \sim 7.78(\mathrm{~m}, 1 \mathrm{H}, \mathrm{PyH}), 7.66$ (d, J=8.1 Hz, 1H, РyH), $7.44(\mathrm{~s}, 1 \mathrm{H}, \mathrm{C}=\mathrm{CH}), 7.22(\mathrm{~d}, J=9.0 \mathrm{~Hz}, 2 \mathrm{H}, \mathrm{ArH})$, $7.15 \sim 7.19$ (m, 1H, PyH), 6.87 (d, $J=9.0 \mathrm{~Hz}, 2 \mathrm{H}, \mathrm{ArH})$, $5.10\left(\mathrm{~s}, 2 \mathrm{H}, \mathrm{CH}_{2}\right), 2.52\left(\mathrm{~s}, 3 \mathrm{H}, \mathrm{CH}_{3}\right) ;{ }^{13} \mathrm{C} \mathrm{NMR}\left(\mathrm{CDCl}_{3}, 75\right.$ MHz) $\delta: 155.7,152.9,150.2,149.1,148.7,147.6,141.3$, $140.4,140.1,138.4,136.7,129.7,128.6,122.3,116.9$, 116.3, 115.7, 103.1, 67.6, 15.2. Anal. calcd for $\mathrm{C}_{20} \mathrm{H}_{15} \mathrm{Cl}_{2-}$ $\mathrm{N}_{5} \mathrm{O}_{2} \mathrm{~S}$ : C 52.18, H 3.28, N 15.21; found C 52.32, H 3.20, $\mathrm{N} 15.37$.

1-(2-吡啶基)-3-甲基-5-(6-氯-3-甲基苯氧基)吡唑-4甲酰基- $O$-[(2-氯噻唑-5-基)甲基]肜(7c): 黄色油状物, 收率 61.2\%. ${ }^{1} \mathrm{H} \mathrm{NMR}\left(\mathrm{CDCl}_{3}, 300 \mathrm{MHz}\right) \delta: 8.39(\mathrm{~d}, J=$ $3.3 \mathrm{~Hz}, 1 \mathrm{H}, \mathrm{PyH}), 7.85(\mathrm{~s}, 1 \mathrm{H}, \mathrm{CH}=\mathrm{N}), 7.73 \sim 7.78(\mathrm{~m}$, 1H, PyH), 7.65 (d, J=8.1 Hz, 1H, PyH), 7.44 (s, 1H, C= $\mathrm{CH}), 7.15 \sim 7.22$ (m, 2H, PyH, ArH), 6.81 (s, 1H, ArH), $6.71(\mathrm{~d}, J=8.4 \mathrm{~Hz}, 1 \mathrm{H}, \mathrm{ArH}), 5.11\left(\mathrm{~s}, 2 \mathrm{H}, \mathrm{CH}_{2}\right), 2.53$ (s, $\left.3 \mathrm{H}, \mathrm{CH}_{3}\right), 2.29\left(\mathrm{~s}, 3 \mathrm{H}, \mathrm{ArCH}_{3}\right) ;{ }^{13} \mathrm{C} \mathrm{NMR}\left(\mathrm{CDCl}_{3}, 75\right.$ $\mathrm{MHz}) \delta: 155.5,152.9,150.2,148.8,147.8,141.4,140.4$, $138.4,137.8,136.8,130.0,128.9,122.3,117.8,115.8$, 114.2, 113.5, 103.0, 67.6, 20.3, 15.3. Anal. calcd for $\mathrm{C}_{21} \mathrm{H}_{17} \mathrm{Cl}_{2} \mathrm{~N}_{5} \mathrm{O}_{2} \mathrm{~S}$ : C 53.17, $\mathrm{H} 3.61, \mathrm{~N} 14.76$; found $\mathrm{C} 53.36$, H 3.50, N 14.62.

1-(2-吡啶基)-3-甲基-5-(1H-吡唑基)吡唑-4-甲酰基$O$-[(2-氯噻唑-5-基) 甲基]肜(7d): 淡黄色固体, 收率 65.4\%. m.p. 96 98 ${ }^{\circ} \mathrm{C}$; ${ }^{1} \mathrm{H}$ NMR $\left(\mathrm{CDCl}_{3}, 300 \mathrm{MHz}\right) \delta$ : $8.28(\mathrm{~d}, J=4.2 \mathrm{~Hz}, 1 \mathrm{H}, \mathrm{PyH}), 7.84(\mathrm{~s}, 1 \mathrm{H}, \mathrm{CH}=\mathrm{N})$, $7.72 \sim 7.76$ (m, 2H, PyH, Pyrazole-H), 7.64 (d, $J=7.2 \mathrm{~Hz}$, $1 \mathrm{H}, \mathrm{PyH}), 7.48$ (s, 1H, C=CH), $7.39(\mathrm{~d}, J=8.4 \mathrm{~Hz}, 1 \mathrm{H}$, Pyrazole-H), $7.17 \sim 7.21$ (m, 1H, PyH), 6.44 (d, $J=1.8 \mathrm{~Hz}$, 
1H, Pyrazole-H), 5.17 (s, 2H, $\left.\mathrm{CH}_{2}\right), 2.57$ (s, 3H, $\left.\mathrm{CH}_{3}\right) ;{ }^{13} \mathrm{C}$ NMR $\left(\mathrm{CDCl}_{3}, 75 \mathrm{MHz}\right) \delta: 152.9,150.5,149.0,148.4$, $142.2,142.0,140.5,138.5,137.7,136.7,133.4,122.7$, $116.8,110.8,107.5,67.7,15.0$. Anal. calcd for $\mathrm{C}_{17} \mathrm{H}_{14} \mathrm{Cl}-$ $\mathrm{N}_{7} \mathrm{OS}$ : C 51.06, H 3.53, N 24.52; found C 51.22, H 3.37, N 24.71 .

1-(2-吡定基)-3-甲基-5-(1H-1,2,4-三唑基)吡唑-4-甲 酰基- $O$-[(2-氯噻唑-5-基)甲基]肜(7e)：白色固体，收率 63.8\%. m.p. $140 \sim 142{ }^{\circ} \mathrm{C} ;{ }^{1} \mathrm{H}$ NMR $\left(\mathrm{CDCl}_{3}, 300 \mathrm{MHz}\right) \delta$ : 8.51 (s, 1H, Triazole-H), 8.15 (d, $J=4.2 \mathrm{~Hz}, 1 \mathrm{H}, \mathrm{PyH})$, $8.11(\mathrm{~s}, 1 \mathrm{H}$, Triazole-H), $7.83(\mathrm{~s}, 1 \mathrm{H}, \mathrm{CH}=\mathrm{N}), 7.74 \sim 7.80$ $(\mathrm{m}, 2 \mathrm{H}, \mathrm{PyH}), 7.48(\mathrm{~s}, 1 \mathrm{H}, \mathrm{C}=\mathrm{CH}), 7.16 \sim 7.20(\mathrm{~m}, 1 \mathrm{H}$, $\mathrm{PyH}), 5.16\left(\mathrm{~s}, 2 \mathrm{H}, \mathrm{CH}_{2}\right), 2.55\left(\mathrm{~s}, 3 \mathrm{H}, \mathrm{CH}_{3}\right) ;{ }^{13} \mathrm{C} \mathrm{NMR}$ $\left(\mathrm{CDCl}_{3}, 75 \mathrm{MHz}\right) \delta: 153.0,152.5,150.8,149.0,148.1$, $147.1,141.1,140.6,138.7,136.4,133.2,122.7,116.2$, 112.1, 67.9, 14.6. Anal. calcd for $\mathrm{C}_{16} \mathrm{H}_{13} \mathrm{ClN}_{8} \mathrm{OS}$ : C 47.94, H 3.27, N 27.95; found C 47.76, H 3.42, N 27.78.

1-(2-吡啶基)-3-甲基-5-(1H-哌啶基)吡唑-4-甲酰基$O$-[(2-氯噻唑-5-基)甲基]肜(7f): 黄色固体, 收率 67.5\%. m.p. 91 93 ${ }^{\circ} \mathrm{C} ;{ }^{1} \mathrm{H}$ NMR $\left(\mathrm{CDCl}_{3}, 300 \mathrm{MHz}\right) \delta: 8.55$ (d, $J=4.5 \mathrm{~Hz}, 1 \mathrm{H}, \mathrm{PyH}), 8.17(\mathrm{~s}, 1 \mathrm{H}, \mathrm{CH}=\mathrm{N}), 7.78 \sim 7.84$ (m, 1H, PyH), 7.66 (d, J=8.1 Hz, 1H, PyH), 7.51 (s, 1H, $\mathrm{C}=\mathrm{CH}), 7.23 \sim 7.28(\mathrm{~m}, 1 \mathrm{H}, \mathrm{PyH}), 5.21\left(\mathrm{~s}, 2 \mathrm{H}, \mathrm{CH}_{2}\right)$, $3.02\left(\mathrm{~s}, 4 \mathrm{H}, 2 \times \mathrm{CH}_{2}\right), 2.43\left(\mathrm{~s}, 3 \mathrm{H}, \mathrm{CH}_{3}\right), 1.53(\mathrm{~s}, 6 \mathrm{H}$, $\left.3 \times \mathrm{CH}_{2}\right) ;{ }^{13} \mathrm{C} \mathrm{NMR}\left(\mathrm{CDCl}_{3}, 75 \mathrm{MHz}\right) \delta: 152.7,152.0$, $151.2,149.2$, 148.6, 144.0, 140.2, 138.0, 137.3, 122.2, $118.4,105.8,67.5,52.0,26.2,23.9,15.1$. Anal. calcd for $\mathrm{C}_{19} \mathrm{H}_{21} \mathrm{ClN}_{6} \mathrm{OS}$ : C 54.73, H 5.08, N 20.16; found C 54.87, H 5.20, N 20.33.

1-(2-吡啶基)-3-甲基-5-(1H-吗啉基)吡唑-4-甲酰基$O$-[(2-氯噻唑-5-基)甲基]肜 $(7 \mathrm{~g})$ ：淡黄色固体，收率 64.1\%. m.p. $76 \sim 78{ }^{\circ} \mathrm{C}$; ${ }^{1} \mathrm{H}$ NMR $\left(\mathrm{CDCl}_{3}, 300 \mathrm{MHz}\right) \delta$ : $8.58(\mathrm{~d}, J=4.8 \mathrm{~Hz}, 1 \mathrm{H}, \mathrm{PyH}), 8.19(\mathrm{~s}, 1 \mathrm{H}, \mathrm{CH}=\mathrm{N})$, $7.99 \sim 8.04(\mathrm{~m}, 1 \mathrm{H}, \mathrm{PyH}), 7.75(\mathrm{~s}, 1 \mathrm{H}, \mathrm{C}=\mathrm{CH}), 7.52(\mathrm{~d}$, $J=7.5 \mathrm{~Hz}, 1 \mathrm{H}, \mathrm{PyH}), 7.44 \sim 7.48(\mathrm{~m}, 1 \mathrm{H}, \mathrm{PyH}), 5.29$ (s, $\left.2 \mathrm{H}, \mathrm{CH}_{2}\right), 3.58\left(\mathrm{~s}, 4 \mathrm{H}, 2 \times \mathrm{CH}_{2}\right), 3.00\left(\mathrm{~s}, 4 \mathrm{H}, 2 \times \mathrm{CH}_{2}\right)$, $2.33\left(\mathrm{~s}, 3 \mathrm{H}, \mathrm{CH}_{3}\right) ;{ }^{13} \mathrm{C} \mathrm{NMR}\left(\mathrm{CDCl}_{3}, 75 \mathrm{MHz}\right) \delta: 151.5$, $150.9,149.3,148.2,147.9,143.5,140.8,138.9,137.7$, $122.9,118.5,105.8,66.8,66.4,50.2,14.4$. Anal. calcd for $\mathrm{C}_{18} \mathrm{H}_{19} \mathrm{ClN}_{6} \mathrm{O}_{2} \mathrm{~S}$ : C 51.61, H 4.57, N 20.06; found C 51.52, $\mathrm{H} 4.42$, N 20.21 .

\section{2 结果与讨论}

\section{1 波谱解析}

分别以目标化合物 $7 \mathbf{b}$ 和 $7 \mathbf{e}$ 的 ${ }^{1} \mathrm{H}$ NMR 和 ${ }^{13} \mathrm{C} N M R$
数据为例进行说明. 对于化合物 $7 \mathbf{b}$, 化学位移在 $\delta 8.36$ 处的双重峰为吡啶环 3-位碳原子上 $\mathrm{H}$ 的质子信号峰; $\delta$ 7.86 处的单峰为 $\mathrm{CH}=\mathrm{N}$ 上 $\mathrm{H}$ 的质子信号峰; $\delta$ 7.72 7.78 间的多重峰为吡定环 5-位碳原子上 $\mathrm{H}$ 的质子信号 峰; $\delta 7.66$ 处的双重峰为吡啶环 6-位碳原子上 $\mathrm{H}$ 的质子 信号峰; $\delta 7.44$ 处的单峰为噻唑环上 $\mathrm{H}$ 的质子信号峰; $\delta$ 7.22 处的双重峰为苯环 3,5-位碳原子上 $\mathrm{H}$ 的质子信号 峰; $\delta 7.15 \sim 7.19$ 间的多重峰为吡啶环 4-位碳原子上 $\mathrm{H}$ 的质子信号峰; $\delta 6.87$ 处的双重峰为苯环 2,6-位碳原子 上 $\mathrm{H}$ 的质子信号峰; $\delta 5.10$ 处的单峰为与噻唑环相连的 亚甲基上 $\mathrm{H}$ 的质子信号峰; 在 $\delta 2.52$ 处的单峰为吡唑环 3-位甲基上 $\mathrm{H}$ 的质子信号峰; $\delta$ 在 152.9 处的峰为 $\mathrm{CH}=$ $\mathrm{N}$ 上碳原子的吸收峰; $\delta 67.6$ 处的峰为与噻唑环相连的 亚甲基碳原子的吸收峰. 对于化合物 7e, 化学位移在 $\delta$ 8.51 处的单峰为三唑环 5-位碳原子上 $\mathrm{H}$ 的质子信号峰; $\delta$ 8.15 处的双重峰为吡啶环 3-位碳原子上 $\mathrm{H}$ 的质子信号 峰; $\delta 8.11$ 处的单峰为三唑环 3-位碳原子上 $\mathrm{H}$ 的质子信 号峰; $\delta 7.83$ 处的单峰为 $\mathrm{CH}=\mathrm{N}$ 上 $\mathrm{H}$ 的质子信号峰; $\delta$ 7.74 7.80 间的多重峰为吡啶环上 5,6-位碳原子上 $\mathrm{H}$ 的 质子信号峰; $\delta 7.48$ 处的单峰为噻唑环上 $\mathrm{H}$ 的质子信号 峰; $\delta 7.16 \sim 7.20$ 间的多重峰为吡啶环 4-位碳原子上 $\mathrm{H}$ 的质子信号峰; $\delta 5.16$ 处的单峰为与噻唑环相连的亚甲 基上 $\mathrm{H}$ 的质子信号峰; 在 $\delta 2.55$ 处的单峰为吡唑环 3位甲基上 $\mathrm{H}$ 的质子信号峰; $\delta$ 在 153.0 处的峰为 $\mathrm{CH}=\mathrm{N}$ 上碳原子的吸收峰; $\delta 67.9$ 处的峰为与噻唑环相连的亚 甲基碳原子的吸收峰.

\section{2 生物活性}

参照文献[26]中试验方法, 测定了目标化合物对小 麦赤霉、番茄早疫、花生褐斑、苹果轮纹和黄瓜枯萎五 种菌体的杀菌活性，测试浓度为 $50 \mu \mathrm{g} / \mathrm{mL}$; 根据文献 [27]中试验方法，测定了标题化合物的植物生长调节活 性, 测试浓度为 $10 \mu \mathrm{g} / \mathrm{mL}$. 杀菌活性试验结果(表 1)表 明，在浓度为 $50 \mu \mathrm{g} / \mathrm{mL}$ 时，部分化合物显示出一定的杀 菌活性(与对照药 Triadimefon 相比), 但并无明显的规 律. 其中化合物 7e 对小麦赤霉的抑制率为 $62.5 \%$; 化合 物 7c 对花生褐斑的抑制率为 $55.3 \%$; 此外，化合物 7f 对黄瓜枯萎的抑制率为 $47.2 \%$. 植物生长调节活性测试 结果(表 1)表明，某些标题化合物对黄瓜子叶生根具有 一定的促进作用，其中化合物 7c 活性相对较高(与对照 药 Triadimefon 相比), 其促进率为 $126.4 \%$. 此外, 根据 文献[28]中试验方法, 测定了化合物对蚕豆蚜、朱砂叶 螨和南方粘虫的杀虫活性, 测试浓度为 $500 \mu \mathrm{g} / \mathrm{mL}$. 杀 虫活性测试结果显示，大多数化合物不具有杀虫活性， 仅化合物 7c 对蚕豆蚜表现出 $47.8 \%$ 的杀死率. 
表 1 目标化合物 7 的杀菌活性和植物生长调节活性 ${ }^{a}$

Table 1 Fungicidal and plant growth gegulatory activities of the title compounds 7

\begin{tabular}{|c|c|c|c|c|c|c|}
\hline \multirow{2}{*}{ 化合物 } & \multicolumn{5}{|c|}{ 杀菌活性/\% } & \multirow{2}{*}{ 植物生长调节活性/\% } \\
\hline & 小麦赤霉 & 番茄早疫 & 花生褐斑 & 苹果轮纹 & 黄瓜枯萎 & \\
\hline $7 \mathbf{a}$ & 13.3 & 22.0 & 0 & 0 & 0 & +36.7 \\
\hline $7 b$ & 14.5 & 0 & 24.6 & 0 & 28.2 & -11.2 \\
\hline $7 c$ & 0 & 20.0 & 55.3 & 20.3 & 13.9 & +126.4 \\
\hline $7 d$ & 0 & 15.3 & 29.3 & 0 & 0 & -37.8 \\
\hline $7 e$ & 62.5 & 23.7 & 21.8 & 0 & 15.6 & +32.1 \\
\hline $7 f$ & 26.3 & 13.5 & 0 & 11.8 & 47.2 & -22.3 \\
\hline $7 \mathrm{~g}$ & 0 & 0 & 0 & 20.0 & 0 & +56.0 \\
\hline Triadimefon & 72.8 & 66.3 & 73.7 & 85.3 & 81.2 & +80.7 \\
\hline
\end{tabular}

${ }^{a}$ 对照药 Triadimefon: 1-(4-氯苯氧基)-3,3-二甲基-1-(1H-1,2,4-三唑-1-基)-2-丁酮.

\section{3 结论}

通过多步反应合成了一系列新型含噻唑环的吡唑 肜醚衍生物. 初步的生物活性测试结果表明, 部分标题 化合物具有一定的杀菌、杀虫或植物生长调节活性, 为 探索新型农药提供了一定的理论依据.

\section{References}

[1] Liu, Y.; Ren, J.; Jin, G. Y. Chin. J. Pestic. Sci. 2001, 3, 12 (in Chinese).

(刘荣, 任军, 金桂玉, 农药学学报, 2001, 3, 12.)

[2] Zhao, P. L.; Wang, L.; Zhu, X. L.; Huang, X. Q.; Zhan, C. G.; Wu, J. W.; Yang, G. F. J. Am. Chem. Soc. 2010, 132, 185.

[3] Liu, X. Y.; Kan, D. L.; Ding, X. C. Acta Chim. Sinica 2011, 69, 1445 (in Chinese) (刘训悦, 阙登蕾, 丁兴成, 化学学报, 2011, 69, 1445.)

[4] Zhang, Q. L.; Yuan, Z. L.; Zhang, Y. Q.; Zhu, B. X. Acta Chim. Sinica 2012, 70, 357 (in Chinese). (张奇龙, 袁泽利, 张云黔, 朱必学, 化学学报, 2012, 70, 357.)

[5] Li, Y. X.; Ling, L.; Chen, Q. Chin. J. Org. Chem. 2011, 31, 306 (in Chinese). (李元祥, 林龙, 陈琼, 有机化学, 2011, 31, 306.)

[6] Chen, L.; Ou, X. M.; Mao, C. H.; Shang, J.; Huang, R. Q.; Bi, F. C.; Wang, Q. M. Bioorg. Med. Chem. 2007, 15, 3678.

[7] Sun, Y. F.; Li, Y. Q.; Ling, Y.; Yu, H. L.; Yang, S. X.; Yang, X. L. Chin. J. Org. Chem. 2011, 31, 1425 (in Chinese).

(孙玉风, 李永强, 凌云, 宇红莲, 杨绍祥, 杨新玲, 有机化学, 2011, 31, 1425.)

[8] An, Y.; Wei, W.; Mou, P. P.; Jia, Jin. Y.; Lü, J. Z.; Chen, X. Chin. J. Org. Chem. 2010, 30, 1726 (in Chinese). (安悦, 魏魏, 牟萍萍, 贾金英, 吕建州, 陈欣, 有机化学, 2010, 30, 1726.)

[9] Balbi, A.; Anzaldi, M.; Macciò, C.; Aiello, C.; Mazzei, M.; Gangemi, R.; Castagnola, P.; Miele, M.; Rosano, C.; Viale, M. Eur. J. Med. Chem. 2011, 46, 5293.

[10] Kang, T. N.; Ling, Y.; Rui, C. H.; Yang, X. L.; Fan, X. L.; Chen, F. H. Chin. J. Org. Chem. 2008, 28, 617 (in Chinese). (康铁牛, 凌云, 茌昌辉, 杨新玲, 范贤林, 陈馥衡, 有机化学, 2008, 28, 617.)

[11] Takano, M.; Enomoto, M.; Saito, K.; Kizawa, S. EP 683160, 1995 [Chem. Abstr. 1995, 124, 202283].

[12] Kim, D. S.; Chun, S. J.; Jeon, J. J.; Lee, S. W.; Joe, G. H. Pest
Manage. Sci. 2004, 60, 1007.

[13] Huang, W.; Yang, G. F. Bioorg. Med. Chem. 2006, 14, 8280.

[14] Rai, N. P.; Venu, T. D.; Manuprasad, B. K.; Shashikanth, S.; Arunachalam, P. N.; Firdouse, A. Chem. Biol. Drug Des 2010, 75, 400.

[15] Aberle, N.; Catimel, J.; Nice, E. C.; Watson, K. G. Bioorg. Med. Chem. Lett. 2007, 17, 3741.

[16] Li, Y. J.; Li, C. Y.; Jin, K.; Sun, S. Q.; Zhou, X. X. Acta Chim. Sinica 2012, 70, 151 (in Chinese).

(李英俊, 李春燕, 靳焜, 孙淑琴, 周晓霞, 化学学报, 2012, 70, 151.)

[17] Yokota, T.; Mikata, K.; Nagasaki, H.; Ohta, K. J. Agric. Food Chem. 2003, 51, 7066.

[18] Wang, M.; Zhang, Y. B. Modern Agrochem. 2003, 2, 36 (in Chinese).

(王敏, 张一宾, 现代农药, 2003, 2, 36.)

[19] Li, N.; Wei, X.; Fan, H. T.; Bi, L.; Li, X. Y.; Yang, S.; Hu, D. Y.; Song, B. A. Chin. J. Org. Chem. 2011, 31, 1300 (in Chinese).

(李娜, 魏学, 范会涛, 毕亮, 李向阳, 杨松, 胡德禹, 宋宝安, 有机化学, 2011, 31, 1300.)

[20] Li, Y.; Zhang, H. Q.; Liu, J.; Yang, X. P.; Liu, Z. J. J. Agric. Food Chem. 2006, 54, 3636.

[21] Luo, Y. P.; Gong, Q.; Chen, Q.; Yang, G. F. Chin. J. Org. Chem. 2008, 28, 1561 (in Chinese).

(骆炎平, 龚青, 陈琼, 杨光富, 有机化学, 2008, 28, 1561.)

[22] Jiang, L. L.; Chen, C. N.; Zhou, Y. F.; Chen, Q.; Yang, G. F. Chin. J. Org. Chem. 2009, 29, 1392 (in Chinese).

(江黎黎, 陈超南, 周延菲, 陈琼, 杨光富, 有机化学, 2009, 29, 1392.)

[23] Cingolani, A.; Marchetti, F.; Pettinari, C.; Pettinari, R.; Skelton, B. W.; White, A. H. Inorg. Chem. 2004, 43, 4387.

[24] Becher, J.; Olesen, P. H.; Knudsen, N. A.; Toftlund, H. Sulfur Lett. 1986, 4,175

[25] Dai, H.; Liu, J. B.; Miao, W. K.; Wu, S. S.; Qin, X.; Zhang, X.; Wang, T. T.; Fang, J. X. Chin. J. Org. Chem. 2011, 31, 1662 (in Chinese).

(戴红, 刘建兵, 苗文科, 吴珊珊, 秦雪, 张欣, 王婷婷, 方建新, 有机化学, 2011, 31, 1662.)

[26] Funaki, Y.; Ishiguri, Y.; Kato, T.; Tanaka, S. J. Pestic. Sci. 1984, 9 , 229.

[27] Demuner, A. J.; Barbosa, L. C. A.; Veloso, D. P. J. Agric. Food Chem. 1998, 46, 1173.

[28] Luo, Y. P.; Yang, G. F. Bioorg. Med. Chem. 2007, 15, 1716. 